\title{
Multiple Quantum Spin-Echo Spectroscopy
}

\author{
G. Bodenhausen, * R. L. Vold, and R. R. Vold \\ Department of Chemistry, University of California-San Diego, La Jolla, California 92093
}

Received February 16, 1979

\begin{abstract}
The generation of nuclear magnetic double quantum echoes and their detection by two-dimensional Fourier transform spectroscopy is reported for partially aligned onedeuteron systems in a nematic liquid crystal. Phase-cycling techniques which suppress detection of unwanted coherences have been developed, and the transverse decay rates of double quantum coherences are found to agree quantitatively with relaxation results obtained by more conventional methods.
\end{abstract}

\section{INTRODUCTION}

Detailed investigations of nuclear spin relaxation in multilevel systems are often carried out with the aim of determining experimental values for some set of relevant spectral densities of motion (1-3). For isotropic fluids the resulting information has proved useful for separating relaxation mechanisms and for characterizing anisotropy of molecular reorientation.

Standard techniques for measuring spin-lattice $\left(R_{1}\right)$ and spin-spin $\left(R_{2}\right)$ relaxation rates in coupled systems are, however, not always sufficient to determine a complete set of spectral density parameters. This situation is particularly common for anisotropic liquid crystalline systems where the complicated motional spectrum spans a wide range of frequencies (4-10). In this context, recent developments in two-dimensional Fourier transform techniques (11-13) and multiple quantum spectroscopy $(11,14-16)$ present exciting opportunities for gathering novel information about relaxation mechanisms and molecular motion. The utility of double and zero quantum coherence decay rates has been demonstrated in a study of a weakly coupled proton $\mathrm{AB}$ spin system subject to relaxation by paramagnetic agents (17). Studies of relaxation in partially ordered deuteron spin systems have demonstrated the feasibility of using double quantum linewidths (18) and have shown the need for such measurements on multispin systems (19) if a complete analysis of motional parameters is desired.

So far, experimental studies have been confined to systems where the natural multiple quantum linewidth is a significant fraction of the total linewidth. In many cases of practical interest, this pleasant situation does not occur, because $n$ quantum linewidths are broadened by $n$ times the normal field inhomogeneity. Furthermore, multiple quantum lineshapes can be distorted by coherence transfer echoes $(14,20)$.

* Present address: Francis Bitter National Magnet Laboratory, Massachusetts Institute of Technology, Cambridge, Massachusetts 02139. 
Both these limitations can be overcome by using refocusing techniques and monitoring multiple quantum echoes $(17,21)$ or by multiple quantum spin-locking procedures (15).

In this paper we are concerned specifically with accurate measurements of double quantum decay rates of deuterium in partially ordered fluid phases. Procedures for excitation and detection of double quantum echoes are discussed, including phasecycled pulse sequences designed to cancel artifacts arising from unwanted single quantum coherence and imperfect pulses. The effect of a single refocusing pulse applied in the middle of the evolution period is considered in detail. Selective detection schemes developed by Wokaun and Ernst (22) are extended to account for the phase of the refocusing pulse and a phase-cycled pulse sequence capable of providing accurate double quantum spin-echo linewidths is presented. Various experimental techniques are illustrated using $\mathrm{CDCl}_{3}$ in nematic liquid crystalline solvents, and extensions to more complex spin systems are discussed.

\section{DENSITY MATRIX DESCRIPTION OF COHERENCE TRANSFER}

Multiple quantum coherence exists in a spin system if the density matrix possesses nonzero off-diagonal elements between eigenstates differing in magnetic quantum number by $\Delta m \neq \pm 1$. Such coherent states can be established by a variety of techniques including a weak "double quantum" pulse applied to an equilibrium system $(15)$ or a strong $(\pi / 2)(11)$ or weak $(14,16)$ pulse applied to a spin system in a nonequilibrium state of either the first or the second kind (23). For excitation of deuterium double quantum coherence in solids or liquid crystals any of these methods may be used. In this section we confine our attention to excitation by means of strong pulses, which may be represented by simple rotation operators in closed analytical form.

In general, the time evolution of the spin density operator $\sigma(t)$ is given by

$$
\sigma(t)=\exp (-i \mathscr{H} t) \sigma(0) \exp (i \mathscr{H} t)
$$

where $\mathscr{H}$ is the effective Hamiltonian operating in the time interval 0 to $t$ and is time independent in an appropriate rotating frame. Our restriction to strong pulses permits Eq. [1] to be simplified:

$$
\sigma\left(t^{+}\right)=R(\alpha, \varphi) \sigma\left(t^{-}\right) R^{-1}(\alpha, \varphi),
$$

where $t^{-}$and $t^{+}$are instants of time just before and after the pulse of flip angle $\alpha$. The phase $\varphi$ is referred to the $x$ axis of the rotating frame. In the eigenstate representation of the static Hamiltonian $\mathscr{H}_{0}$, individual density matrix elements $\sigma_{p q}$ correspond either to eigenstate populations $(p=q)$ or to coherence between such states $(p \neq q)$. Utilizing the unitary property of the rotation operator $R_{s q}^{-1}(\alpha, \phi)=$ $R_{q s}^{*}(\alpha, \phi)$ we may write

$$
\sigma_{p q}\left(t^{+}\right)=\sum_{r s} R_{r r}(\alpha, \phi) \sigma_{r s}\left(t^{-}\right) R_{q s}^{*}(\alpha, \phi) .
$$

This equation represents the transfer of coherence from $\sigma_{r s}\left(t^{-}\right)$before the pulse to $\sigma_{p q}\left(t^{+}\right)$after the pulse, and the probability of coherence transfer is given by the 
magnitude of $R_{p r} R_{q s}^{*}$ (11). By examining the individual terms in Eq. [3] (or elements of the supermatrix $R \otimes R$ in the Liouville representation), detailed pathways of coherence transfer can be discerned. Thus, the transfer equation is a powerful tool in multiple quantum spectroscopy, especially if an analytical expression for $R(\alpha, \phi)$ is available. Other workers $(15,24,25)$ have avoided the matrix element evaluations implicit in Eq. [3] by expanding Eq. [1] or Eq. [2] in fictitious spin or single transition operator representations and using commutator algebra, but for the systems considered here the direct evaluation of Eq. [3] is straightforward. Moreover, extensions to situations where numerical methods are nceded to evaluatc $R(\alpha, \phi)$ arc conceptually simple.

\section{EXCITATION AND DETECTION OF DOUBLE QUANTUM COHERENCE FOR A SINGLE SPIN $I=1$}

In this section we consider the three-level system of a single spin $I=1$-the simplest case for which double quantum coherence can occur. It is desirable to obtain an explicit expression for $R(\alpha, \phi)$ in the eigenstate basis of the static Hamiltonian

$$
\mathscr{H}_{0}=-\Delta \omega I_{z}+\frac{1}{3} \omega_{\mathrm{Q}}\left(3 I_{z}^{2}-\mathbf{I} \cdot \mathbf{I}\right) \text {. }
$$

Here $\Delta \omega=\left(\omega_{0}-\omega\right)$ is the offset of the pulse carrier frequency $\omega$ from exact resonance $\omega_{0}$, and $2 \omega_{\mathrm{O}}$ is the residual quadrupolar splitting observed in a partially ordered medium. The rotation operator is given by

$$
\begin{aligned}
R(\alpha, \phi) & =\exp \left\{i \alpha\left(I_{x} \cos \phi+I_{y} \sin \phi\right)\right\} \\
& =\exp \left(-i \phi I_{z}\right) \exp \left(i \alpha I_{x}\right) \exp \left(i \phi I_{z}\right) .
\end{aligned}
$$

For a single spin $I=1$, the angular momentum operators are represented by $3 \times 3$ matrices. In the eigenbasis of Eq. [4], $\exp \left(-i \phi I_{z}\right)$ is a diagonal matrix, and simple recursive methods $(26)$ can be used to evaluate $\exp \left(i \alpha I_{x}\right)$. Thus, we obtain $R(\alpha, \phi)=$

$$
\left[\begin{array}{c:c:c}
\frac{1}{2}(\cos \alpha+1) & \left(i / 2^{1 / 2}\right) \sin \alpha \exp (-i \phi) & \frac{1}{2}(\cos \alpha+1) \exp (-2 i \phi) \\
\left(i / 2^{1 / 2}\right) \sin \alpha \exp (i \phi) & \cos \alpha & \left(i / 2^{1 / 2}\right) \sin \alpha \exp (-i \phi) \\
\frac{1}{2}(\cos \alpha+1) \exp (2 i \phi) & \left(i / 2^{1 / 2}\right) \sin \alpha \exp (i \phi) & \frac{1}{2}(\cos \alpha+1)
\end{array}\right] .
$$

Consider the pulse sequence $90_{x}^{\circ}-\tau_{-} 90_{x}^{\circ}$ used by Aue et al. (11) and Drobny et al. (27) to generate multiple quantum coherence. The first pulse acts on an equilibrium system, for which the single quantum population differences are equal (and unity by convention). Using Eq. [3] with $\sigma\left(0^{-}\right)=I_{z}$ and Eq. [6] with $\alpha=90^{\circ}$ and $\phi=0^{\circ}$, we see that the first pulse generates only single quantum coherences $\sigma_{12}\left(0^{+}\right)=\sigma_{23}\left(0^{+}\right)=$ $-i / 2^{1 / 2}$, aligned along the $y$ axis of the rotating frame. Double quantum coherence $\sigma_{13}$ remains zero, and the populations $\sigma_{p p}\left(0^{+}\right)$are equal. At the end of the $\tau$ interval, assuming that no relaxation has occurred, the single quantum magnetizations will have gained phase angles $\omega_{12} \tau$ and $\omega_{23} \tau$, respectively, where $\omega_{12}$ and $\omega_{23}$ are the 
transition frequencies between eigenstates of Eq. [4]:

$$
\begin{aligned}
& \omega_{12}=\omega_{\mathrm{Q}}-\Delta \omega, \\
& \omega_{23}=\omega_{\mathrm{Q}}-\Delta \omega .
\end{aligned}
$$

Thus, we obtain

$$
\sigma\left(\tau^{-}\right)=-i / 2^{1 / 2}\left[\begin{array}{c:c:c}
0 & \exp \left(i \tau\left(\Delta \omega-\omega_{\mathrm{Q}}\right)\right) & 0 \\
-\exp \left(-i \tau\left(\Delta \omega-\omega_{\mathrm{Q}}\right)\right) & 0 & \exp \left(i \tau\left(\Delta \omega+\omega_{\mathrm{Q}}\right)\right) \\
0 & -\exp \left(-i \tau\left(\Delta \omega+\omega_{\mathrm{Q}}\right)\right) & 0
\end{array}\right] \text {. }
$$

The effect of the second pulse can be found by inserting Eq. [8] into Eq. [3], using Eq. [6] as before with $\alpha=90^{\circ}, \phi=0^{\circ}$. The result is

$$
\begin{aligned}
\sigma_{13}\left(\tau^{+}\right) & =\frac{-i}{2\left(2^{1 / 2}\right)}\left(\sigma_{12}\left(\tau^{-}\right)+\sigma_{21}\left(\tau^{-}\right)\right)+\frac{i}{2\left(2^{1 / 2}\right)}\left(\sigma_{23}\left(\tau^{-}\right)+\sigma_{32}\left(\tau^{-}\right)\right), \\
& =i \cos \Delta \omega \tau \sin \omega_{\mathrm{Q}} \tau
\end{aligned}
$$

The quantities $\frac{1}{2}\left(\sigma_{12}\left(\tau^{-}\right)+\sigma_{21}\left(\tau^{-}\right)\right)$and $\frac{1}{2}\left(\sigma_{23}\left(\tau^{-}\right)+\sigma_{32}\left(\tau^{-}\right)\right)$are simply $x$ components of single quantum magnetizations prior to the second $90^{\circ}$ pulse. Equation [9] demonstrates that double quantum excitation is maximized if two criteria are met: (1) The two single quantum magnetizations should be opposite in phase and (2) they should be aligned along the $B_{1}$ vector, i.e., the $x$ axis of the rotating frame. Choosing $\tau=p \pi / 2 \omega_{\mathrm{Q}}$ (for odd integer $p$ ) fulfills the first condition, and adjusting the resonance offset such that $\Delta \omega=2 k \omega_{\mathrm{Q}}$ (for integer $k$ ) fulfills the second condition. This procedure is demonstrated experimentally in Fig. 1.

Following stimulation, the double quantum coherence precesses at frequency $2 \Delta \omega$, independent of $\omega_{\mathrm{Q}}(15)$ :

$$
\sigma_{13}\left(t_{1}\right)=i \cos \Delta \omega \tau \sin \omega_{\mathrm{Q}} \tau \exp \left(-2 i \Delta \omega t_{1}\right) .
$$

Since the coherence cannot be detected directly, a monitoring pulse is applied at the end of the evolution period to induce a transfer of coherence into the observable quantities $\sigma_{12}$ and $\sigma_{23}$. The monitoring pulse, in effect, reverses the transfer accomplished by the second excitation pulse. According to Eqs. [3] and [6], where we assune for the moment that $\phi=0^{\circ}$ for the monitoring pulse, we find

$$
\begin{aligned}
\sigma_{12}\left(t_{1}^{+}\right) & =R_{11} R_{23}^{*} \sigma_{13}\left(t_{1}^{-}\right)+R_{13} R_{21}^{*} \sigma_{31}\left(t_{1}^{-}\right) \\
& =\left(2^{-1 / 2}\right) \sin \alpha\left(\operatorname{Im} \sigma_{13}\left(t_{1}^{-}\right)-i \cos \alpha \operatorname{Re} \sigma_{13}\left(t_{1}^{-}\right)\right), \\
\sigma_{23}\left(t_{1}^{+}\right) & =R_{21} R_{33}^{*} \sigma_{13}\left(t_{1}^{-}\right)+R_{23} R_{31}^{*} \sigma_{31}\left(t_{1}^{-}\right) \\
& =-\left(2^{-1 / 2}\right) \sin \alpha\left(\operatorname{Im} \sigma_{13}\left(t_{1}^{-}\right)-i \cos \alpha \operatorname{Re} \sigma_{13}\left(t_{1}^{-}\right)\right) .
\end{aligned}
$$

It is apparent that the coherence transfer produced by the monitoring pulse is maximized for a flip angle $\alpha=90^{\circ}$. In this case, the phases of $\sigma_{12}\left(t_{1}^{+}\right)$and $\sigma_{23}\left(t_{1}^{+}\right)$are opposite and independent of $t_{1}$. Thus, the double quantum precession gives rise to pure amplitude rather than phase modulation $(11,15)$. 


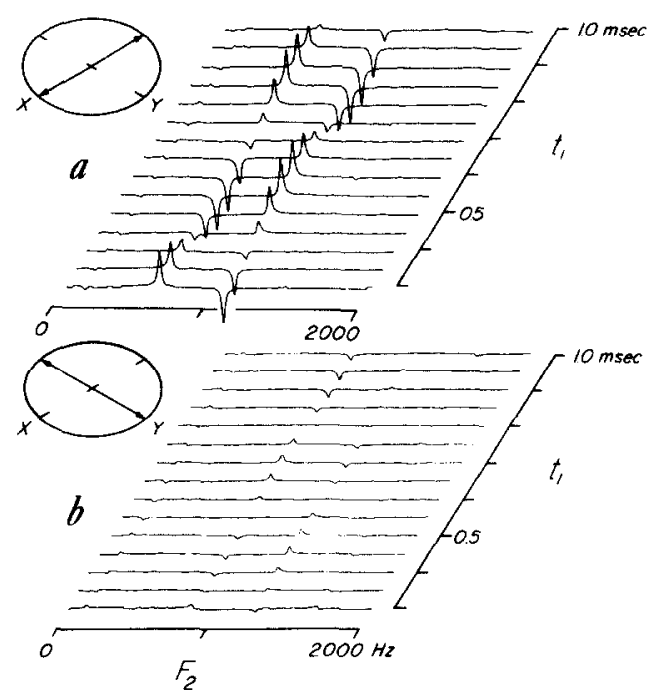

FIG. 1. Excitation of double quantum coherence for a single spin $I=1$ in a nematic liquid crystalline solution using the pulse sequence $90^{\circ}-\tau-90^{\circ}-t_{1}-90^{\circ}-\mathrm{FT}\left(t_{2}\right)$. The amplitude modulation observed in (a) arises from double quantum coherence generated by the second $90^{\circ}$ pulse. For this system the quadrupolar splitting $2 \omega_{\mathrm{Q}}=418 \mathrm{~Hz}$ and the use of $\tau=p \pi / 2 \omega_{\mathrm{Q}}(p=1,3,5, \ldots)$ optimizes the generation of double quantum coherence, when the resonance offset is simultaneously adjusted such that $\Delta \omega=2 k \omega_{\mathbf{Q}}(k=$ $1,2, \ldots$ ). In (a), $\Delta \omega \simeq 4 \omega_{\mathrm{Q}}=812 \mathrm{~Hz}$ such that after $\tau=1.208 \mathrm{msec}$ the single quantum magnetizations are aligned parallel to $B_{1}$ along the $x$ axis of the rotating frame. Conversely in (b) minimum excitation of double quantum coherence is achieved by setting $\Delta \omega \approx 5 \omega_{\mathrm{Q}}=1015 \mathrm{~Hz}$. The sample used in this illustration as well as in Figs. 2 through 4 is ca. $10 \%(w / w)$ poly- $\gamma$-benzyl-L-glutamate (PBLG) in $\mathrm{CDCl}_{3}$ and the temperature was $30^{\circ} \mathrm{C}$. Suppression of single quantum interference was achieved using the phase-cycling techniques described in the text. The deuteron spectra presented here were obtained at 9.2 $\mathrm{MHz}$ on a home-built pulse spectrometer interfaced to a Nicolet 1083 Fourier-transform system. The NTCFT software package was modified to allow execution of 2DFT experiments, while hardware changes in the 293-pulse programmer were relatively minor. A separate rf gate unit, which allows phase-shifting of the rf pulses under software control, has been described elsewhere (43).

If the monitoring pulse has a flip angle $a \neq 90^{\circ}$, then $\sigma_{12}\left(t_{1}^{+}\right)$and $\sigma_{23}\left(t_{1}^{+}\right)$are modulated in phase as well as amplitude. Equations [10] and [11] yield the explicit result,

$$
\begin{aligned}
\sigma_{12}\left(t_{1}^{+}\right) & =-\sigma_{23}\left(t_{1}^{+}\right) \\
& =\left(2^{-1 / 2}\right) \sin \alpha \cos \Delta \omega \tau \sin \omega_{\mathrm{Q}} \tau\left(\cos 2 \Delta \omega t_{1}-i \cos \alpha \sin 2 \Delta \omega t_{1}\right) .
\end{aligned}
$$

Thus, if subsequent evolution of $\sigma_{12}$ and $\sigma_{23}$ is observed and Fourier-transformed to yield a data array $S\left(t_{1}, F_{2}\right)$, spectra obtained for successive increments in $t_{1}$ will exhibit a phase shift of $\tan ^{-1}\left(\cos \alpha \tan 2 \Delta \omega t_{1}\right)$. Pure amplitude modulation (obtained with $\alpha=90^{\circ}$ ) yields double absorption mode signals upon the second Fourier transformation, which are more convenient for linewidth measurements.

\section{PHASE-SHIFTED EXCITATION AND SELECTIVE DETECTION}

Consider the double-pulse excitation sequences $90^{\circ}{ }_{\phi}-\tau-90^{\circ}{ }_{\phi}$, where the phase $\phi$ can be shifted at will. Using methods illustrated in the previous section, it is easy to 
show that

$$
\sigma_{13}\left(\tau^{+}\right)=i \cos \Delta \omega \tau \sin \omega_{\mathrm{Q}} \tau \exp (-2 i \phi) .
$$

Comparison with Eq. [9] reveals that shifting the phase of the excitation pulses by $\phi$ results in a double quantum phase shift $2 \phi(11,15)$. This property forms the basis for selective detection of multiple quantum coherence of specified order (22).

During the evolution period following a double $90^{\circ}$ pulse excitation, populations will recover and the monitoring pulse will transfer the population differences into the single quantum manifold, producing a signal which grows toward the equilibrium magnetization as $t_{1} \rightarrow \infty$. This undesired signal can be suppressed by co-addition of signals from two successive experiments in which the phase of the monitoring pulse is changed by $180^{\circ}$. In order to avoid simultaneous cancellation of the double quantum signal its phase must also be reversed. This can be accomplished by a $90^{\circ}$ phase shift of both excitation pulses.

In practice, the double-pulse excitation generates both single and double quantum coherence; hence, the signal following the monitoring pulse stems not only from transfer of $\sigma_{13}$, but also from ordinary single quantum magnetization precessing during the evolution period. This effect is reminiscent of nonideal inversion recovery experiments, where spurious transverse magnetization generated by an imperfect inversion pulse may lead to distorted signals for short values of $t_{1}(28)$. According to Eq. [13], alternating the phase of both excitation pulses by $180^{\circ}$ leaves the double quantum phase unchanged, while the spurious single quantum contributions will alternate in sign and therefore cancel.

Both forms of single quantum interference mentioned above can be cancelled by adding four transients from successive experiments in which the excitation phase is incremented through $0^{\circ}, 90^{\circ}, 180^{\circ}, 270^{\circ}$, while the monitoring phase alternates $0^{\circ}, 180^{\circ}, 0^{\circ}, 180^{\circ}$. This sequence was in fact used to obtain Fig. 1, where the signals arise solely from transfer of double quantum coherence.

A further example of this procedure is shown in Fig. 2. Here the four-step phase-cycled pulse sequence was used, keeping a fixed, very small $(50-\mu s e c)$ value of $t_{1}$ and incrementing the excitation time $\tau$ in 100 - $\mu$ sec steps. This experiment is useful in practice as a "setup" technique because for vanishingly small $t_{1}$, the excitation and monitoring pulses are necessarily equally efficient. Hence, the optimum value of $\tau$ corresponds simply to the largest signal.

\section{REFOCUSING AND DOUBLE QUANTUM SPIN ECHOES}

Using techniques described in the preceding section, it is possible to obtain double quantum spectra which are free from single quantum artifacts, with two-dimensional lineshapes in double absorption mode, well suited to linewidth measurement. Since the double quantum precession frequency is independent of $\omega_{\mathrm{Q}}(15)$, the double quantum linewidth is not subject to broadening from a distribution of static order parameters caused, for instance, by temperature gradients. Thus, if the true double quantum decay rate is short enough, simple linewidth measurements can provide accurate relaxation information (18). However, double quantum decay rates are twice as sensitive to static field inhomogeneity as are single quantum decays $(11,15)$. 


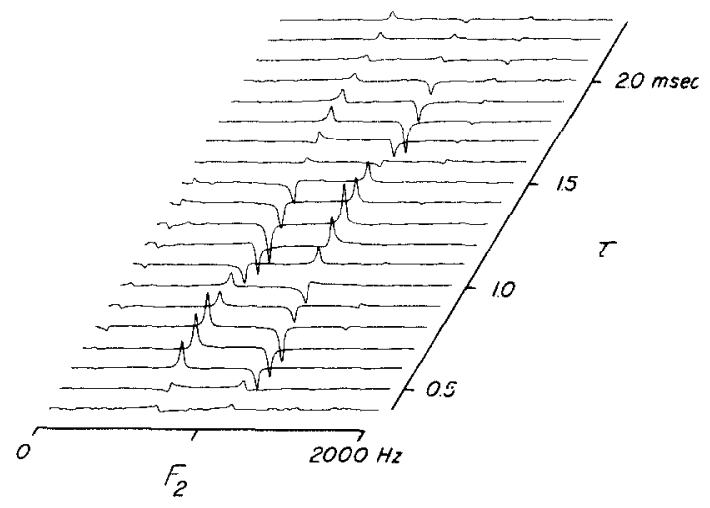

FIG. 2. "Setup" experiment for optimization of double quantum coherence in a $90^{\circ}-\tau-90^{\circ}-t_{1}-90^{\circ}-$ FT $\left(t_{2}\right)$ experiment. A fixed value of $t_{1}\left(50 \mu \mathrm{sec}, \ll(2 \Delta \omega)^{-1}\right)$ was maintained while $\tau$ was incremented in steps of $0.1 \mathrm{msec}$. Signal intensities obey Eq. [10] and the maximum at $1.2 \mathrm{msec}$ corresponds to optimal double quantum excitation. As in Fig. 1, single quantum interference is cancelled in a four-step cycle in which the phase of the first two pulses is stepped through $0^{\circ}, 90^{\circ}, 180^{\circ}, 270^{\circ}$, while the monitoring pulse is alternated between $0^{\circ}$ and $180^{\circ}$.

In addition, phase coherence which is lost during double quantum evolution may be (partially) refocused during single quantum detection, leading to a multiple quantum coherence transfer echo $(14,20)$ and consequent lineshape distortion. These effects render double quantum linewidths unreliable for use in relaxation studies if the true decay times are longer than a few milliseconds. Clearly, refocusing techniques are needed.

Consider the effect of a $180^{\circ}$ pulse of phase $\phi$, applied at $t_{1} / 2$ in the middle of the evolution period. From Eqs. [3] and [6] we obtain

$$
\begin{aligned}
& \sigma_{12}\left(t_{1}^{+} / 2\right)=\sigma_{23}^{*}\left(t_{1}^{-} / 2\right) \exp (-2 i \phi), \\
& \sigma_{23}\left(t_{1}^{+} / 2\right)=\sigma_{12}^{*}\left(t_{1}^{-} / 2\right) \exp (-2 i \phi), \\
& \sigma_{13}\left(t_{1}^{+} / 2\right)=\sigma_{13}^{*}\left(t_{1}^{-} / 2\right) \exp (-4 i \phi) .
\end{aligned}
$$

Expressing the static field inhomogeneity across the sample by an offset $\Delta \omega(\mathbf{r})$, the phase coherence at time $t_{1}$ is given by

$$
\begin{aligned}
\sigma_{12}\left(t_{1}\right) & =\left\{\sigma_{23}(0) \exp \left(\frac{-i t_{1}}{2}\left[\omega_{23}+\Delta \omega(\mathbf{r})\right]\right)\right\}^{*} \exp \left(\frac{-i t_{1}}{2}\left[\omega_{12}+\Delta \omega(\mathbf{r})\right]\right) \cdot \exp (-2 i \phi) \\
& =\sigma_{23}^{*}(0) \exp \left(\frac{-i t_{1}}{2}\left[\omega_{12}-\omega_{23}\right]\right) \cdot \exp (-2 i \phi) \\
\sigma_{23}\left(t_{1}\right) & =\sigma_{12}^{*}(0) \exp \left(\frac{i t_{1}}{2}\left[\omega_{12}-\omega_{23}\right]\right) \cdot \exp (-2 i \phi) \\
\sigma_{13}\left(t_{1}\right) & =\sigma_{13}^{*}(0) \exp (-4 i \phi)
\end{aligned}
$$

Thus, dephasing due to field inhomogeneity is refocused for both single and double quantum signals. Since the pulse interconverts $\sigma_{12}$ and $\sigma_{23}$, the single quantum 
echoes are phase-modulated in a manner similar to those in scalar coupled spin- $-\frac{1}{2}$ systems (29). The $180^{\circ}$ pulse does not mix single quantum with double quantum signals (17), and from Eq. [15c] it is apparent that all double quantum precession is refocused.

In accord with the well-known result of Meiboom and Gill (30), a $90^{\circ}$ phase shift of the refocusing pulse reverses the phase of the single quantum echoes. If a phase reversal of the double quantum echo is desired, the phase of the refocusing pulse must be advanced by $45^{\circ}$. A $90^{\circ}$ phase shift leaves the phase of the double quantum echo unchanged. These properties are useful for designing refocusing pulse sequences which suppress artifacts.

In practice, refocusing pulses are bound to deviate from the ideal flip angle of $180^{\circ}$ in at least some parts of the sample. To appreciate the consequences, it is useful to classify the density matrix elements $\sigma_{p q}(n)$ according to order $n=m_{p}-m_{q}$, where $m_{p}$ and $m_{q}$ are magnetic quantum numbers of the eigenstates $|p\rangle$ and $|q\rangle$. In single quantum two-dimensional spin-echo experiments, an ideal refocusing pulse reverses the order $\sigma_{r s}( \pm 1) \rightarrow \sigma_{p q}(\mp 1)$. Imperfect pulses, acting on partially saturated populations, generate new transverse magnetizations $\sigma_{r r}(0) \rightarrow \sigma_{p q}( \pm 1)$. Such processes give rise to extra "phantom" lines in two-dimensional spectra (12). In addition, a fraction of the magnetization is unaffected by an imperfect pulse and simply continues its precession. Such artifacts are known as "ghosts" (12). Both types of artifacts are effectively suppressed by a four-step "exorcycle" sequence (31).

In multiple quantum experiments, the situation is considerably more complicated since an imperfect pulse causes coherence transfer between all orders $n$. Such transfer processes can be distinguished on the basis of their response of rf phase shifts (22):

$$
\begin{aligned}
\sigma_{p q}\left(n^{+}\right) & =\sum_{r s} R_{p r}(\alpha, \phi) \sigma_{r s}\left(n^{-}\right) R_{q s}^{*}(\alpha, \phi), \\
& =\sum_{r s} R_{p r}(\alpha, 0) \sigma_{r s}\left(n^{-}\right) R_{q s}^{*}(\alpha, 0) \exp \left(-i\left(n^{+}-n^{-}\right) \phi\right) .
\end{aligned}
$$

Here + and - refer as usual to times just after and just before the pulse. The phase shift $4 \phi$ experienced by a double quantum coherence upon ideal refocusing is unique and sets this type of transfer apart from all other nonideal forms of coherence transfer. Thus, advancing the phase of the refocusing pulse by $45^{\circ}$ causes the double quantum echo to reverse in phase, while all other transfer processes experience phase shifts of $0,45^{\circ}, 90^{\circ}$, or $135^{\circ}$.

An experiment which cancels all forms of undesirable coherence transfer in systems with one or two deuterons consists of a sequence of 16 transients (hex adecacycle). The phase of both excitation pulses is maintained at zero for the first eight transients, and shifted to $180^{\circ}$ for the last eight. The phase of the refocusing pulse is advanced in regular increments $0,45^{\circ}, 90^{\circ}, 135^{\circ}, \ldots, 315^{\circ}$, while the monitoring pulse alternates between 0 and $180^{\circ}$. This sequence achieves cancellation of the single quantum artifacts discussed in the preceding section, as well as echoes arising from coherences of all odd orders and even orders $n=4 p, p=1,2,3, \ldots$.

The success of this procedure is illustrated in Fig. 3. Each data array $S\left(t_{1}, F_{2}\right)$ consists of $2000-\mathrm{Hz}$ regions of the usual spectrum, for the first 20 values of $t_{1}$ in a 


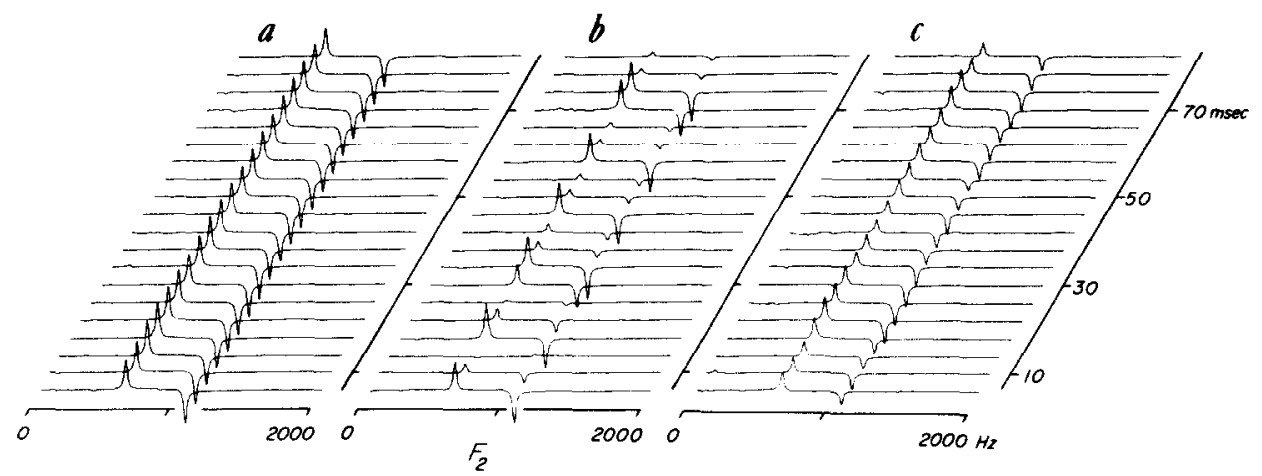

Fig. 3. Double quantum spin echoes. Each data array $S\left(t_{1}, F_{2}\right)$ consists of $2000-\mathrm{Hz}$ regions of the $F_{2}$ domain, for the first 20 values of $t_{1}$ in the double quantum spin-echo pulse sequence $90^{\circ}-\tau-90^{\circ}-t_{1} / 2-\alpha-$ $t_{1} / 2-90^{\circ}-\mathrm{FT}\left(t_{2}\right)$. The vertical scale is the same for all three data arrays, and in all cases $\tau=1.2 \mathrm{msec}$ and $\Delta t_{1}=4 \mathrm{msec}$. In (a) the phase-cycling scheme illustrated in Fig. 1 was used, except that a $180^{\circ}$ refocusing pulse at $t_{1} / 2$ has been inserted, with the same phase as the excitation. The absence of amplitude modulation shows that refocusing of double quantum precession has occurred. In (b) the refocusing pulse was purposely misset to $\alpha \sim 90^{\circ}$, and the resulting modulation arises from nonideal coherence transfer produced by this pulse. In (c), which has the same vertical scale as (a) and (b), $\alpha$ is still misset to $90^{\circ}$ but the 16 -step hexcycle sequence described in the text was used to suppress undesirable coherence transfer. The total time $t_{1}$ covered in these experiments is too short to show significant transverse double quantum relaxation.

two-dimensional spin-echo experiment $\left(90^{\circ}-\tau-90^{\circ}-t_{1} / 2-\alpha-t_{1} / 2-90^{\circ}\right.$-acquisition). In Fig. 3a, the four-phase cycle discussed in the preceding section was used, except that a refocusing pulse with $\alpha=180^{\circ}$ was inserted at $t_{1} / 2$ with the same phase as the excitation. Note the absence of amplitude modulation-the double quantum precession has been refocused. In Fig. $3 b$, the same pulse sequence was used but the refocusing pulse was purposely misset to $\alpha \simeq 90^{\circ}$, and nonideal coherence transfer severely modulates the echo train. In Fig. 3c the 16-step "hexcycle" was used, with $\alpha$ still misset to $90^{\circ}$. Undesirable coherence transfers, amounting to roughly half the total signal amplitude, have been effectively suppressed. The decay of the remaining signals provides an accurate measure of the double quantum relaxation rate. The full amplitude of double quantum echo signals is regained by setting $\alpha=180^{\circ}$.

In general, it is unfortunate that the refocusing pulse suppresses double quantum precession, because the resulting two-dimensional double quantum spin-echo spectrum yields peaks at $F_{1}=0$. Experience with two-dimensional spectroscopy has shown that modulated interferograms yield spectra which are more suitable for accurate linewidth measurements in the $F_{1}$ domain (32). Furthermore, it may be desirable to separate multiple quantum echoes of higher order which occur in more complicated spin systems (27). A simple device, which solves both problems at once, in effect forces all echoes to be modulated. This scheme requires the transmitter phase to be advanced as $t_{1}$ is incremented and should not be confused with the phase-cycling techniques discussed so far, which differentiate between subsequent transients recorded for a given $t_{1}$. By advancing the phase of both excitation and refocusing pulses for the $k$ th $t_{1}$ value by $k \pi / l$, where $l$ is an arbitrary integer, the phase of an $n$-quantum echo signal is advanced by $n \pi k / l$. For example, with $l=6$ the 


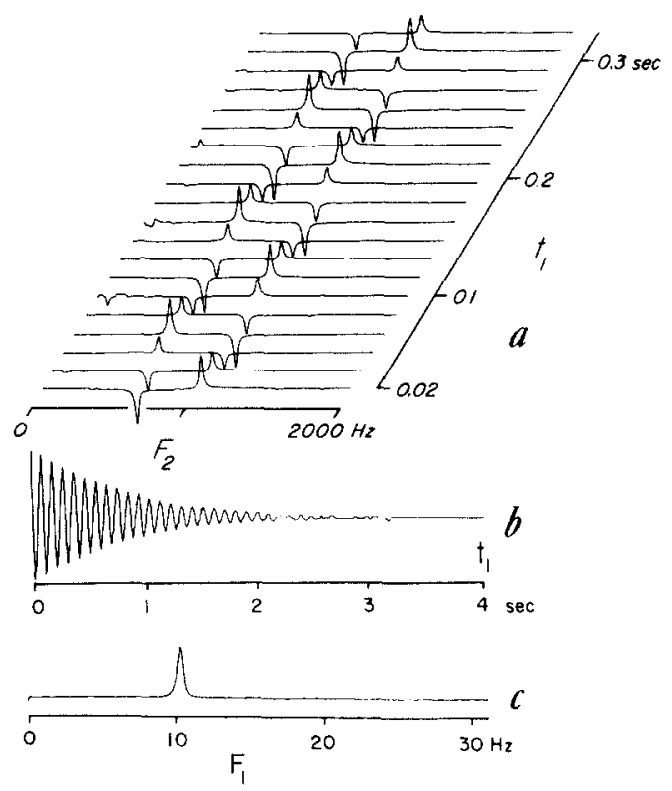

FIG. 4. Double quantum echo experiment with phase advancement. Excitation and detection of the double quantum echo were performed using the hexcycle sequence described in the text. In addition, the phase of both excitation pulses was advanced by $30^{\circ}$ for each value of $t_{1}$. The resulting amplitude modulation is shown in (a). In (b), the result of sampling the top of the right-hand resonance is shown: This is the double quantum echo decay, which in the absence of phase advancement would appear as a simple unmodulated exponential. Fourier transformation of (b) yields the spectrum in (c), where the double quantum coherence occurs at one-third the spectral width. The linewidth yields a rate $R_{1313}=1.33 \mathrm{sec}^{-1}$ for the transverse relaxation of the double quantum coherence of $10 \%(\mathrm{w} / \mathrm{w}) \mathrm{PBLG}$ in $\mathrm{CDCl}_{3}$.

phase of the double quantum echo advances through $60^{\circ}$ for each $t_{1}$ increment. This leads to modulated echoes as shown in Fig. 4, with subsequent Fourier transformation giving a peak displaced from $F_{1}=0$ by a fraction $n / l$ (e.g., $\frac{2}{6}$ ) of the spectral width. Subsequent to completion of this work, we learned that this incremental phase advancement technique had been independently developed by Drobny et al. (27).

\section{DOUBLE QUANTUM DECAY RATES FOR $I=1$ SYSTEMS}

For a single deuteron the double quantum coherence decays exponentially with a rate constant given by the Redfield relaxation matrix element $R_{1313}$. If the dominant relaxation mechanism is of quadrupolar origin, one obtains $(18,33)$

$$
R_{1313}=\frac{3 \pi^{2}}{2}\left(e^{2} q Q / h\right)^{2}\left\{J_{1}\left(\omega_{0}\right)+2 J_{2}\left(2 \omega_{0}\right)\right\} .
$$

Here $\left(e^{2} q O / h\right)$ is the deuterium quadrupole coupling constant and $J_{1}\left(\omega_{0}\right)$ and $J_{2}\left(2 \omega_{0}\right)$ are spectral density functions, normalized (34) such that in the "white spectrum" limit for an isotropic medium, $J_{1}\left(\omega_{0}\right)=J_{2}\left(2 \omega_{0}\right)=\frac{1}{5} \tau_{\mathrm{R}}$, where $\tau_{\mathrm{R}}$ is the rotational correlation time. From repeated measurements of the double quantum 
spin-echo linewidth we obtain $R_{1313}=1.33 \pm 0.06 \mathrm{sec}^{-1}$ for our liquid crystalline solution of $10 \%(\mathrm{w} / \mathrm{w}) \mathrm{PBLG}$ in deuterochloroform at $35^{\circ} \mathrm{C}$. It is of interest to compare this decay rate with the spin-lattice relaxation rate $R_{1}=1.17 \pm 0.01 \mathrm{sec}^{-1}$, which we determined by inversion recovery using nonselective pulses. The latter value agrees well with previous determinations (35). For quadrupolar relaxation, $R_{1}$ is given by

$$
R_{1}=\frac{3 \pi^{2}}{2}\left(e^{2} q Q / h\right)^{2}\left\{J_{1}\left(\omega_{0}\right)+4 J_{2}\left(2 \omega_{0}\right)\right\}
$$

$J_{1}\left(\omega_{0}\right)$ and $J_{2}\left(2 \omega_{0}\right)$ are necessarily positive, and therefore, $R_{1313} / R_{1}$ must be less than or equal to unity according to Eqs. [17] and [18]. The observed double quantum decay rate is greater than $R_{1}$ by a factor of $1.14 \pm 0.05$, which is inconsistent with Eqs. [17] and [18].

It is evident that processes exist which dephase the double quantum coherence but which are not included in Eq. [17]. Contributions from diffusion through (possibly local) regions of inhomogeneous field can be dismissed; they would yield echo amplitudes proportional to $\exp \left(-t_{1}^{3}\right)$, whereas the observed decay (Fig. 4) is exponential. The double quantum decay rate of $1.33 \pm 0.06 \mathrm{sec}^{-1}$ was determined using a variety of excitation and detection procedures, and the same value of $R_{1313}$ was observed with multiple refocusing of the double quantum coherence using a series of $180^{\circ}$ pulses, in analogy with Carr-Purcell method B (36). The most plausible explanation for the fast double quantum decay rate involves chemical exchange. In the simplest (and no doubt oversimplified) version of this picture, one imagines two sites for partially ordered deuterochloroform, differing in residual quadrupolar splitting by $\delta \omega_{\mathrm{Q}}$ and in resonance frequency by $\delta \omega_{0}$. Exchange between such sites modulates both $\omega_{\mathrm{O}}$ and $\omega_{0}$ in a random fashion but since the double quantum precession frequency is independent of $\omega_{Q}$, dephasing results solely from the modulation of $\omega_{0}$. By analogy with single quantum spin-echo decays $(37,38)$, an approximate expression for the contribution to the double quantum decay rate is $\left(2 \delta \omega_{0}\right)^{2} / 8 k$, where $k$ is the average exchange rate between equally populated sites. Chemical shift differences as small as 0.1 to $0.2 \mathrm{~Hz}$, combined with exchange rates of $\sim 1 \mathrm{sec}^{-1}$, suffice to account for our observations. Extensive sample rotation measurements $(39,40)$ indicate that distinct sites certainly exist in liquid crystalline PBLG solutions, and very small chemical shift differences are not unreasonable. It is worth noting that the parameters used here to account for the double quantum decay rate predict negligible contributions to the high-resolution lineshape. It appears that double quantum spin echoes can be useful in investigating slow dynamical processes.

We have measured double quantum spin-echo decays for a $10 \mathrm{~mol} \%$ solution of deuterochloroform in Merck Phase V. For this sample the quadrupolar splitting $2 \omega_{\mathrm{Q}}=29 \mathrm{kHz}$ is large enough that all pulses are certainly far from ideal. Nevertheless, we obtained $R_{1313}=13.7 \pm 0.4 \mathrm{sec}^{-1}$, in close agreement with the value $13.3 \pm$ $0.7 \mathrm{sec}^{-1}$ calculated from Eqs. [17] and [18] using spectral density parameters determined previously (18). We conclude that double quantum spin-echo linewidths measured using the 16-step phase-cycled pulse sequence are largely free of pulse artifacts. 


\section{MULTISPIN SYSTEMS}

The analysis presented in the preceding sections for a single spin $I=1$ can be extended in straightforward fashion to multispin systems. For a set of $\boldsymbol{N}$ deuterons, $R(\alpha, \phi)$ may be found by forming the direct product of $N 3 \times 3$ matrices of the form of Eq. [6], followed by a similarity transformation to the eigenstate basis. It is somewhat simpler to compute $R(\alpha, 0)$ in this manner, and to account for the rf phase using Eq. [16]. For $N>2$, the resulting expressions are cumbersome and best handled by a computer. Nevertheless, certain general features can be discerned as described below.

When applied to a system of $N$ deuterons in a partially ordered mesophase, the $90^{\circ}-\tau-90^{\circ}$ excitation sequence will generate all orders of multiple quantum coherence up to and including $2 N$. It is convenient to classify the coherence on the basis of subsequent precession frequency: Class 1 consists of all coherences (offdiagonal density matrix elements) with precession frequencies dependent only on resonance offset, class 2 is comprised of coherences with precession determined by resonance offset and ${ }^{2} \mathrm{H}-{ }^{2} \mathrm{H}$ dipolar interactions, while class 3 coherences precess at rates determined, at least in part, by quadrupolar interactions. Classes 2 and 3 are unique to systems of two or more spins. For example, in the two-deuteron system (19) there are six double quantum coherences (two of each class), two class 3 triple quantum coherences, and one class 1 four-quantum coherence.

Multiple quantum coherences of different classes can be operationally distinguished on the basis of their "resonance" frequencies in a two-dimensional spin-echo experiment. Since Zeeman interactions are refocused by the $180^{\circ}$ pulse while dipolar and quadrupolar interactions are not, class 1 coherences give rise to signals at $F_{1}=0$ (unless phase advancement is used). For deuteron systems, in which dipolar interactions are typically less than a few hundred hertz while quadrupolar interactions are normally several kilohertz, class 3 coherences will usually be aliased in the $F_{1}$ domain while class 1 coherences are not. More fundamentally, there is a one-to-one correspondence between eigenstates which define class 1 coherence and eigenstates which are interconverted by spin inversion. Consequences of this fact will be explored in a future publication.

For purposes of spin-echo studies of multiple quantum relaxation it is advisable to avoid exciting class 3 coherence. A small fractional inhomogeneity in liquid crystalline order (e.g., resulting from minor thermal gradients) leads to a large dispersion of quadrupolar precession frequencies. Since this is not refocused by the $180^{\circ}$ pulse, class 3 coherence will give rise to spuriously wide lines. Analogous dispersion of class 2 coherence is much smaller since dipolar interactions are small.

In cases where the relaxation information inherent in class 3 coherence is needed, refocusing may be achieved with $90^{\circ}$ pulses (41). However, a $90^{\circ}$ refocusing pulse will produce a variety of unwanted coherence transfer phenomena which would have to be suppressed by appropriate phase-cycling schemes. Equation [16], which applies equally well to single and multiple spin systems, is useful for designing such sequences.

In multispin systems it is likely that several multiple quantum coherences may precess at a common frequency. In perfect analogy with degenerate transverse magnetizations in scalar coupled systems (42), cross-relaxation then gives rise to 
multiple exponential decays. This feature, as well as the averaging effect (2) of the $180^{\circ}$ refocusing pulse, must be considered when analyzing the spin-echo lineshapes. These and other aspects of using multiple quantum spin echoes in relaxation studies will be discussed in a future publication.

\section{SUMMARY}

The experiments described here have demonstrated the feasibility of generating and detecting double quantum echoes for partially ordered deuteron spin systems. Echoes of all orders of coherence are generated by the application of a strong $180^{\circ}$ pulse in the middle of the evolution period, and phase-cycling schemes have been devised to suppress undesirable coherence transfers, while retaining echo signals of specified order. We have found a 16-step "hexcycle" particularly useful for observation of double quantum echoes which are free of artifacts.

The echo experiments presented here rely on the use of one $180^{\circ}$ refocusing pulse only; in other words, they correspond to the well-known Carr-Purcell Method A spin echo experiment (36). Use of this method in transverse relaxation measurements is limited to situations where the natural relaxation time is short enough that diffusion effects are negligible. This limitation becomes even more stringent for spin echoes of higher order $(n)$ since the diffusion term grows with $n^{2}$. For the deuteron systems studied here diffusion effects appear negligible, since magnetic field homogeneity is good and natural relaxation times are short. Two-dimensional multiple quantum spin-echo spectra thus provide a promising new source of relaxation information. For proton spin systems in nonviscous liquids multiple pulse refocusing schemes will be required. Such methods may be useful provided phase-cycling schemes for suppression of various artifacts can be devised. From a more positive point of view one may envision the use of higher-order spin echoes in studies of translational diffusion.

\section{ACKNOWLEDGMENTS}

This work was supported by grants from the National Science Foundation (CHE 76-03890 and CHE 77-22164) and the U.S. Public Health Service (RR-00708).

\section{REFERENCES}

1. L. G. Werbelow AND D. M. Grant, Adv. Magn. Reson. 9, 190 (1977).

2. R. L. VOLd AND R. R. VOld, Progr. NMR Spectr. 12, 79 (1978).

3. W. M. M. J. BovÉE, Mol. Phys. 29, 1673 (1975).

4. P. L. Nordio And P. Busolin, J. Chem. Phys. 55, 5485 (1971).

5. C. F. Polnaszek And J. H. Freed, J. Phys. Chem. 79, 2283 (1975).

6. J. P. JACOBSEN AND K. SCHAUMBURG, J. Magn. Reson. 24, 173 (1976).

7. P. Ukleja, J. Pirs, And J. W. Doane, Phys. Rev. A 14, 414 (1976).

8. L. G. Wfrkfi ow, D. M. Grant, E. P. Black, and J. M. Courtieu, J. Chem. Phys, 69, 2407 (1978).

9. R. R. Vold And R. L. Vold, J. Chem. Phys. 71, 1508 (1979).

10. R. L. Vold, R. R. Vold, R. Poupko, AND G. Bodenhausen, J. Magn. Reson. in press.

11. W. P. Aue, E. BARtholdi, AND R. R. ERnst, J. Chem. Phys. 64, 2229 (1976). 
12. G. Bodenhausen, R. Freeman, R. Niedermeyer, AND D. L. Turner, J. Magn. Reson. 26, 133 (1977).

13. G. A. MorRis AND R. Freeman, Bull. Magn. Reson. 1, 1 (1979).

14. H. Hatanaka, T. Terao, and T. Hashi, J. Phys. Soc. Japan 39, 835 (1975).

15. S. Vega And A. Pines, J. Chem. Phys. 66, 5624 (1977).

16. M. E. Stoll, A. J. Vega, And R. W. Vaughan, J. Chem. Phys. 67, 2029 (1977).

17. A. WOKAUN AND R. R. ERNST, Mol. Phys. 36, 317 (1978).

18. G. Bodenhausen, N. M. SzeVerenyi, R. L. Vold, And R. R. Vold, J. Am. Chem. Soc. 100, $6265(1978)$.

19. R. POUPKo, R. L. Vold, AND R. R. Vold, J. Magn. Reson. 34, 67 (1979).

20. A. A. Maudsley, A. Wokaun, And R. R. Ernst, Chem. Phys. Lett. 55, 9 (1978).

21. H. Hatanaka AND T. Hashi, $J$ Phys. Soc. Japan 39, 1139 (1975).

22. A. Wokaun ANd R. R. ERnst, Chem. Phys. Lett. 52, 407 (1977).

23. S. SCHÄublin, A. HöHENER, AND R. R. ERnSt, J. Magn. Reson. 13, 196 (1974).

24. A. Wokaun ANd R. R. ERnst, J. Chem. Phys. 67, 1752 (1977).

25. S. VEgA, J. Chem. Phys. 68, 5518 (1978).

26. P. L. CORIO, "Structure of High-Resolution NMR Spectra," Appendix III, p. 483, Academic Press, New York, 1966.

27. G. Drobny, A. Pines, S. Sinton, D. Weitekamp, and D. Wemmer, Disc. Faraday Soc., in press.

28. D. E. Demco, P. van Hecke, And J. S. Waugh, J. Magn. Reson. 16, 467 (1974).

29. R. Freeman AND H. D. W. HILl, in "Dynamic Nuclear Magnetic Resonance Spectroscopy (L. M. Jackman and F. A. Cotton, Eds.), p. 131 and references therein, Academic Press, New York, 1975.

30. S. MeIBOOM AND D. Gill, Rev. Sci. Instr. 29, 688 (1958).

31. G. Bodenhausen, R. Freeman, And D. L. Turner, J. Magn. Reson. 27, 511 (1977).

32. G. Bodenhausen AND R. Freeman, J. Magn. Reson. 28, 303 (1977).

33. J. P. JACOBSEN, H. K. BILdsøe, AND K. SchaumbuRG, J. Magn. Reson. 23, 153 (1976).

34. R. R. VOld AND R. L. Vold, J. Chem. Phys. 66, 4018 (1977).

35. B. M. Fung AND T. H. Martin, J. Chem. Phys. 61, 1698 (1974).

36. H. Y. CARr AND E. M. PurCell, Phys. Rev. 94,630 (1954).

37. Z. Luz And S. Meiboom, J. Chem. Phys. 59, 275 (1973).

38. H. S. Gutowsky, R. L. Vold, AND E. J. Wells, J. Chem. Phys. 43, 4107 (1965).

39. R. D. Orwoll and R. L. Vold, J. Am. Chem. Soc. 93, 5335 (1971).

40. R. W. Filas, L. E. Hajdo, And A. C. Eringen, J. Chem. Phys. 61, 3037 (1974).

41. S. B. Ahmad, K. J. Packer, And J. M. Ramsden, Mol. Phys. 33, 857 (1977).

42. A. G. REDFIELD, Adv. Magn. Reson. 1, 1 (1965).

43. G. Bodenhausen, J. Magn. Reson. 34, 357 (1979). 